\title{
Thymectomy and myasthenia gravis: A history of surgical passion and scientific excellence
}

\author{
Joshua R. Sonett, MD, ${ }^{\mathrm{a}, \mathrm{b}}$ Mitchell J. Magee, MD, ${ }^{\mathrm{c}}$ and Lyall Gorenstein, $\mathrm{MD}^{\mathrm{a}, \mathrm{b}}$
}

\footnotetext{
From the ${ }^{\mathrm{a} D i v i s i o n}$ of Cardiothoracic and Vascular Surgery, ${ }^{\mathrm{b}}$ Columbia University Medical Center, New-York Presbyterian Hospital, New York, NY; and ${ }^{\mathrm{c}}$ Medical City Dallas Hospital, Dallas, Tex.

Received for publication Oct 6, 2016; revisions received Dec 5, 2016; accepted for publication Dec 17, 2016; available ahead of print May 4, 2017

Address for reprints: Joshua R. Sonett, MD, Division of Cardiothoracic and Vascular Surgery, Columbia University Medical Center, New-York Presbyterian Hospital, 161 Fort Washington Ave, 3rd Floor, Herbert Irving Pavilion, New York, NY 10032 (E-mail: Js2106@cumc.columbia.edu).

J Thorac Cardiovasc Surg 2017;154:306-9

$0022-5223 / \$ 36.00$

Copyright (c) 2017 by The American Association for Thoracic Surgery

http://dx.doi.org/10.1016/j.jtcvs.2016.12.074
}

The results of the Myasthenia Gravis Thymectomy Trial (MGTX), a randomized prospective trial recently published in the New England Journal of Medicine, determined that thymectomy significantly improves the clinical course of patients with myasthenia gravis (MG). Specifically, thymectomy was associated with improved quantitative neurologic measures of $\mathrm{MG}$, reduced medication requirements to control symptoms, and reduced diseaserelated hospital admissions. ${ }^{1}$ Patients with nonthymomatous MG were randomized prospectively into 2 treatment groups: medical therapy alone or surgery with medical therapy. Led by dedicated visionaries, Drs Fred Jaretzki and John Newsom-Davis, the trial was planned and implemented over 12 years, with 6 years of patient accrual followed by 3 years of surveillance. Unfortunately, both Jaretzki and Newson-Davis died before the completion of the MGTX trial, depriving them the satisfaction of seeing decades of passionate surgical insight, dedication, and debate come to fruition.

The modern investigation of thymectomy in MG began in 1939, when Blalock reported a single patient whose MG dramatically improved after resection of a thymic mass. $^{2}$ In an extensive surgical treatise that reviewed previous pathologic and experimental work, Blalock presented a pathophysiologic theory as to the role of thymectomy in MG. He reviewed 4 patients with MG who underwent surgery specifically to alter the course of the disease. Two patients died, but the symptoms of MG improved in the 2 surviving patients. ${ }^{2,3}$ Blalock admitted openly: "We wish to emphasize again the absence of conclusive proof that the improvement noted in our patient is due to the removal of the tumor from the thymic region.",

Continuing his initial investigation, Blalock performed a series of thymectomies in patients with nonthymomatous $\mathrm{MG}$, attempting to further define the value and role of thymectomy in MG. In 1941, he reported a series of 6 patients who underwent transsternal thymectomy, including a cervical dissection, for nonthymomatous $\mathrm{MG}^{4}$ In his operative technique, he stressed the need for meticulous exploration."4

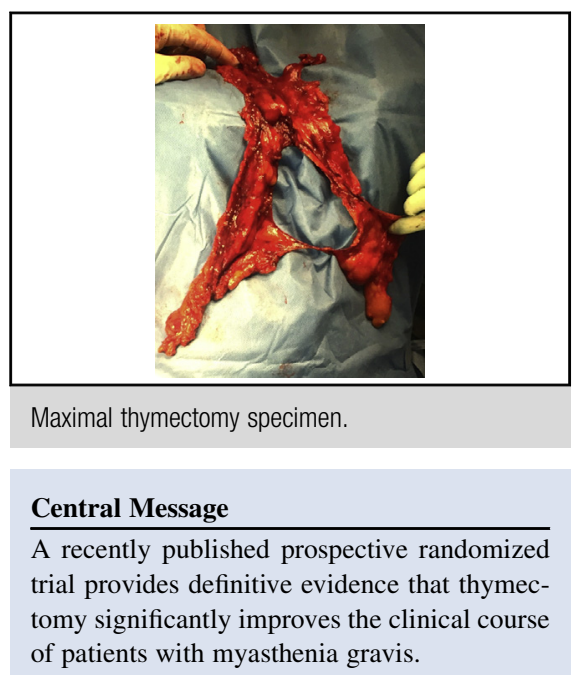

See Editorial Commentary page 310.

See Editorial page 312 .

preoperative and postoperative care of these patients. Three patients did remarkably well and were weaned off medications, whereas 2 improved modestly. With these publications, the role of thymectomy in $\mathrm{MG}$ was established sufficiently to engender generations of surgeons to explore the merits of various thymectomy techniques in the treatment of MG. Laying the groundwork for future surgical controversies, he stated "The present attempt to influence the course of myasthenia gravis differs from those described in that the operation was performed with the deliberate purpose of removing all the thymic tissue by complete

Following these initial reports, multiple nonrandomized surgical series reported benefits of thymectomy, solidifying in the eyes of surgeons and some neurologists the role of thymectomy in $\mathrm{MG}^{5-7}$ Surgical debate moved from the indications for surgery to the most appropriate technique of thymectomy. In 1969, Kirschner and colleagues ${ }^{8}$ revisited Sauerbruch's original cervical surgical approach to thymectomy, publishing a more contemporary series of transcervical thymectomy. The cervical approach in his report included an elective tracheostomy in all patients and descriptive details of using traction to deliver the "well defined thymic tissue from the mediastinal fat." This was particularly relevant at the time, as refinements in medical care of patients with $\mathrm{MG}$ as well as surgical 
and anesthetic technique were still evolving in a way that favored the cervical approach over transsternal thymectomy.

Dr Fred Jaretzki highlighted the divergent techniques and advocated for alternative approaches in a 1977 publication': After attempting the transcervical approach, Dr Jaretzki was convinced that complete extirpation of all viable thymic tissue via the originally described transcervical approach was not ideal and would compromise patient outcomes. Jaretzki demonstrated that pathologically proven anatomic thymic variants and extra-anatomic thymic rests (thymic tissue/cells in mediastinal adipose tissue) and lobes exist in most patients. ${ }^{9}$ Based on these findings, he asserted that a combined transsternal and cervical approach was necessary to attain a radical resection and later published follow-up data using this approach, therein reporting the highest complete response rates for myasthenia. Presentation of these results incited a spirited debate defending the various surgical techniques. ${ }^{9}$

Dr Joel Cooper articulated innovations in the cervical approach used at the University of Toronto that improved exposure and enhanced complete resection of thymic tissue. $\mathrm{He}$ wrote, "We have developed a technique for transcervical thymectomy that allows complete excision under direct vision, avoiding the need for blunt dissection and the incomplete visualization that were associated with this approach. ${ }^{10}$ Describing the newly designed Cooper retractor that elevates the sternum and provides improved visualization, he published results of this modified transcervical approach as identical to the maximal thymectomy of Jaretzki. In a later discussion of his long-term results, Cooper stated that, "If someone told me that you could do a complete clean-out of the mediastinum through a neck incision, I would tell out they were smoking something. But with modification, with the retractor, the headlights and loops...we are accomplishing the same thing." ${ }^{11}$ With improved technology and instrumentation, different surgical camps developed that used rather disparate approaches, but most were convinced that a complete and maximal thymectomy was important to cure patients with MG.

While surgeons debated the approach to thymectomy, primarily with single-series retrospective studies, the medical community retained serious questions about the true value of thymectomy in MG. In 2000, the American Academy of Neurology published an extensive review of the role of thymectomy. ${ }^{12}$ The review concluded that "We cannot determine from the available studies whether the observed association between thymectomy and improved MG outcome was a result of a thymectomy benefit or was merely a result of the multiple differences in baseline characteristics between surgical and non-surgical groups. Based on these findings, we conclude that the benefit of thymectomy in nonthymomatous $\mathrm{MG}$ has not been established conclusively." The manuscript and society called for a prospective study randomizing patients with nonthymomatous MG to medical therapy with and without thymectomy.

The continued need for definitive proof of the surgical effectiveness of thymectomy propelled the Neurologic Division of the National Institutes of Health to fund a prospective study of the role of thymectomy in MG. Planning began in 2001, and the MTGX trial began accruing patients in 2006. ${ }^{1}$ The article most recently published in the New England Journal of Medicine involved 68 centers (32 centers in the United States), 18 countries, 6 continents, and randomized 126 patients with acetylcholine antibody-positive, nonthymomatous MG to prednisone therapy alone versus prednisone therapy with extended transsternal thymectomy. Trial inclusion criteria were strict and included duration of MG of less than 5 years, an age of 18 to 65 years, a serum acetylcholine receptor antibody level of more than $1.0 \mathrm{nmol} / \mathrm{L}$, and a Myasthenia Gravis Foundation of America clinical classification of II to IV (class I indicates weakness only in ocular muscles, class II mild generalized disease, class III moderate generalized disease, class IV severe generalized disease, and class $\mathrm{V}$ a crisis requiring intubation). The study was powered to measure a $30 \%$ difference in outcomes. The dual primary outcomes were clinical status as measured by the time-weighted average Quantitative Myasthenia Gravis score and the time-weighted average required dose of prednisone over a 3-year period. Secondary outcomes included serious adverse events, days of hospitalization over the 3-year period, and quality-oflife surveys. ${ }^{1}$

To insure that the only surgical variable was the thymectomy, and not the type of thymectomy, an extended transsternal thymectomy was mandated. All surgeons were required to adhere to the specified technique as demonstrated in a mandatory training session that included video observation and testing as part of the protocol. All operative notes, pathology reports, and specimen pictures were reviewed centrally. Follow-up of the patients was performed in a blinded assessment by neurologists, and all patients were prescribed mock turtlenecks to hide surgical scars. Stringent protocols were developed to assure stable and consistent medical therapy, with prednisone dosing and withdrawal strictly prescribed by objective neurologic measures. Prednisone was delivered in special blister packs that were retrieved by study coordinators to assure that prescribed and actual dosing were identical.

The results from this study unequivocally prove that extended transsternal thymectomy improves clinical outcomes of patients with generalized MG. Patients who were randomized to transsternal thymectomy had significantly improved symptoms of $\mathrm{MG}$, including an 
average quantitative myasthenia score of 6.15 versus 8.99 , a difference of 2.85 in favor of the thymectomy group $(P>.0001)$. The lower the quantitative myasthenia score, the better the patient is symptomatically, and a difference of this magnitude is considered clinically significant. Importantly, a significantly lower dose of prednisone was needed to attain improved neurologic status (44 $\mathrm{mg}$ vs $60 \mathrm{mg} ; P<.001)$. Treatment-associated complications were not significantly different between the 2 treatment groups over a period of 3 years. However, other findings that favored thymectomy over prednisone alone included the time-weighted average score on the Myasthenia Gravis Activities of Daily Living scale (2.24 vs $3.41, P=.008)$, azathioprine use $(17 \%$ vs $48 \%$ of participants, $P<.001$ ), and the percentage of patients who had minimal-manifestation status at month $36(67 \%$ vs $47 \%, P=.03)$. In addition, hospitalizations during the trial period for myasthenia-related symptoms were significantly lower in the thymectomy group $(9 \%$ vs $37 \%$ ). Interestingly, despite these quantitative results, no difference was seen in the quality-of-life measured surveys.

The study protocol further specified the analysis of 3 subgroups: (1) age at disease onset $<40$ years versus $\geq 40$ years, (2) sex, and (3) previous or no previous glucocorticoids use. The full benefit of thymectomy was confirmed regardless of age; however, the benefit of thymectomy for men and women was not equal. Women in the study had improvements in both measured clinical status and lower prednisone dosing, whereas men only had a significant improvement with regard to lower prednisone dosing. Finally, in terms of previous glucocorticoids use, patients who were prednisone naïve before entering the study were not observed to benefit from thymectomy.

An important ancillary study, Bio-MGTX, was performed simultaneously. The results of this study will examine genomic and proteomic profiles, autoantibody profiles, and histopathology of prospectively collected tissue and blood in an effort to identify possible blood markers predictive of response as well other pathologic and serum prognosticators. Many questions still need to be answered in regard to the role of thymectomy in MG. Possible radiologic predictors of success have not yet been analyzed, and the role of thymectomy in seronegative MG, ocular MG, and elderly patients are yet to be proven definitively. Future studies may be directed at achieving a more rapid and consistent time to a complete symptom response. Data dating back to $1977^{13}$ and replicated in other studies ${ }^{14}$ support high-dose prednisone induction combined with thymectomy as a means to achieve higher complete early remission rates within a much quicker response time (less than a year). That the prednisone-naïve group in the MGTX trial received no significant benefit lends support to the use of induction therapy. Presently, with improved immunosuppressive therapy, more refined or directed induction protocols ( $\mathrm{T}$ - or B-cell directed) may be warranted, particularly if they are based on data from the Bio-MGTX trial.

The debate will likely continue in regard to widespread adoption of extended transsternal maximal thymectomy. What was categorically measured in this trial was the effect of maximal thymectomy, as sternotomy offers no particular independent therapeutic benefit. A maximal thymectomy attempts to remove all tissue in the mediastinum and neck that frequently harbors extra-anatomic thymic lobes and rests of thymic tissue, including tissue lateral to the phrenic nerves and tissue extending to the costophrenic sulcus. ${ }^{15}$ Different techniques to achieve maximal thymectomy are now used commonly, and many surgeons have achieved excellent results with a variety of surgical approaches, including transcervical, subxiphoid, video-assisted thoracic surgery, and robotic thymectomy. ${ }^{16-20}$ Even so, we must remain cognizant of the difficulties in equating techniques, given the wide variance of patients, medical treatments, and outcome measures in most single-series reports. The onus is on the surgical community to prove equivalence of unilateral approaches versus bilateral/cervical approaches that afford direct bilateral dissection.

The importance of the MGTX trial cannot be overemphasized, both for thymectomy and for surgical clinical trials. Despite the widespread belief among surgeons that thymectomy improved the lives and prognosis of many patients with MG, clear proof did not exist, and this created enough doubt to diminish the use of thymectomy in MG. The tightly controlled protocol of this trial enabled the medical and surgical community to definitively answer an important question with a very small cohort of only 126 patients. The study also demonstrated that wide variances in clinical and surgical practices could be controlled within the confines of a prospective trial in multiple institutions across the globe. In the age of large database retrospective analyses that provide valuable but often limited information, we cannot forget the true power of well-designed prospective randomized studies to answer important surgical questions.

The MGTX trial conclusively proved that thymectomy improved clinical outcomes over a 3-year period in patients with nonthymomatous MG. However, additional investigation and refinement in the treatment of MG is still sorely needed. Even when provided the best treatment currently accepted, patients may require 1 to 3 years or more to achieve an optimal response. Our treatment goal in each patient should be complete cure of the disease and to achieve this in a much shorter time frame. Multimodality therapy is the basis of virtually all modern cancer treatment, and the same principles should apply to the treatment of 
MG. Continued progress in the management of MG will require diligent, multidisciplinary teams designing and completing prospective studies like the MGTX. Continued excellence in investigation and innovation as well as passionate debate regarding the best surgical approach will certainly continue and should be relished as drivers of our academic mission.

\section{Conflict of Interest Statement}

Authors have nothing to disclose with regard to commercial support.

\section{References}

1. Wolfe GI, Kaminski HJ, Aban IB, Minisman G, Kuo HC, Marx A, et al. Randomized Trial of Thymectomy in Myasthenia Gravis. N Eng J Med. 2016; 375:511-22.

2. Blalock A, Mason MF, Morgan HJ, Riven SS. Myasthenia gravis and tumors of the thymic region: report of a case in which the tumor was removed. Ann Surg. 1939;110:544-61.

3. Sauerbruch H, Schumacher CB, Roth J. Thymektomie bei einem Fall von Morbus Basedowi mit Myasthenie [Thymectomy in case of disease with myasthenia]. Mitteil Grenzgeb Med Chir. 1913;25:746-65.

4. Blalock A, Harvey AM, Ford FR, Lilienthal JL Jr. The treatment of myasthenia gravis (by) removal of the thymus gland: preliminary report. JAMA. 1941;117: 1529 .

5. Keynes G. Surgery of the thyus gland; second (and third) thoughts. Lancet. 1954; 263:1197-202.

6. Ross RT. Thymectomy in the treatment of myasthenia gravis. Lancet. 1952;262: 785-7.

7. Perlo VP, Poskanzer DC, Schwab RS, Viets HR, Osserman KE, Genkins G. Myasthenia gravis: evaluation of treatment in 1355 patients. Neurology. 1966; 16:431-9.

8. Kirschner P, Osserman KE, Kark AE. Studies in myasthenia gravis: transcervical total thymectomy. JAMA. 1969;209:906-10.
9. Jaretzki A III, Bethea M, Wolff M, Olarte MR, Lovelace RE, Penn AS, et al. A rational approach to total thymectomy in the treatment of myasthenia gravis. Ann Thorac Surg. 1977;24:120-30.

10. Cooper JD, Al-Jilaihawa AN, Pearson G, Humphrey JG, Humphrey E. An improved technique to facilitate transcervical thymectomy for myasthenia gravis. Ann Thorac Surg. 1988;45:242-7.

11. Calhoun RF, Ritter JH, Guthrie TH, Pestronk A, Meyers BF, Patterson GA, et al Results of transcervical thymectomy for myasthenia gravis in 100 consecutive patients. Ann Surg. 1999;230:555-9.

12. Gronseth GS, Barohn RJ. Practice parameter: thymectomy for autoimmune myasthenia gravis (an evidence-based review): report of the Quality Standards of the American Academy of Neurology. Neurology. 2000;55 7-15.

13. Bolooki H, Schwartzman RJ. High-dose steroid for perioperative management of patients with myasthenia gravis undergoing thymectomy. A preliminary report J Thorac Cardiovasc Surg. 1978;75:754-7.

14. Sekine Y, Kawaguchi N, Hamada C, Sekiguchi H, Yasufuku K, Iyoda A, et al. Does perioperative high- dose prednisolone have clinical benefits for generalized myasthenia gravis? Eur J Cardiothorac Surg. 2016;29: 908-13.

15. Sonett JR, Jaretzki A III. Thymectomy for nonthymomatous myasthenia gravis: a critical analysis. Ann N Y Acad Sci. 2008;1132:315-28.

16. Rückert JC, Ismail M, Swierzy M, Sobel H, Rogalla P, Meisel A, et al. Thoracoscopic thymectomy with the da Vinci robotic system for myasthenia gravis. Ann NY Acad Sci. 2008;1132:329-35.

17. Zieliński M, Hauer L, Kuzdzał J, Sośnicki W, Harazda M, Pankowski J, et al Technique of the transcervical-subxiphoid- videothoacoscopic maximal thymectomy. J Minim Access Surg. 2007:3:168-72.

18. Tomulescu V, Sgarbura O, Stanescu C, Campeanu A, Herlea V, Popescu I. Ten year results of thoracoscopic unilateral extended thymectomy performed in nonthymomatous myasthenia gravis. Ann Surg. 2011;254:761-5.

19. Novellino L, Longoni M, Spinelli L, Andretta M, Cozzi M, Faillace G, et al. "Extended" thymectomy, without sternotomy, performed by cervicotomy and thoracoscopic technique in the treatment of myasthenia gravis. Int Surg. 1994 79:378-81.

20. Singh G, Glotzbach J, Costa J, Gorenstein L, Ginsburg M, Sonett JR. Minimally invasive radical thymectomy. Ann Cardiothorac Surg. 2016;5:59-61. 